\title{
Asymmetric sequential Landau-Zener dynamics of Bose-condensed atoms in a cavity
}

\author{
Jiahao Huang (黄嘉豪),${ }^{1,2}$ Pu Gong (龚璞), ${ }^{3}$ Xizhou Qin (秦锡洲), ${ }^{1}$ Honghua Zhong (钟宏华), ${ }^{1,4}$ \\ and Chaohong Lee (李朝红) ${ }^{1,2, *}$ \\ ${ }^{1}$ TianQin Research Center \& School of Physics and Astronomy, Sun Yat-Sen University (Zhuhai Campus), Zhuhai 519082, China \\ ${ }^{2}$ Key Laboratory of Optoelectronic Materials and Technologies, Sun Yat-Sen University (Guangzhou Campus), Guangzhou 510275, China \\ ${ }^{3}$ Department of Physics and Center for Theoretical and Computational Physics, The University of Hong Kong, China \\ ${ }^{4}$ Department of Physics, Jishou University, Jishou 416000, China
}

(Received 17 February 2016; revised manuscript received 5 March 2016; published 15 August 2016)

\begin{abstract}
We explore the asymmetric sequential Landau-Zener (LZ) dynamics in an ensemble of interacting Bosecondensed two-level atoms coupled with a cavity field. Assuming the couplings between all atoms and the cavity field are identical, the interplay between atom-atom interaction and detuning may lead to a series of LZ transitions. Unlike the conventional sequential LZ transitions, which are symmetric to the zero detuning, the LZ transitions of Bose-condensed atoms in a cavity field are asymmetric and sensitively depend on the photon number distribution of the cavity. In LZ processes involving single excitation numbers, both the variance of the relative atom number and the step slope of the sequential population ladder are asymmetric, and the asymmetry becomes more significant for smaller excitation numbers. Furthermore, in LZ processes involving multiple excitation numbers, there may appear asymmetric population ladders with decreasing step heights. During a dynamical LZ process, due to the atom-cavity coupling, the cavity field shows dynamical collapses and revivals. In comparison with the symmetric LZ transitions in a classical field, the asymmetric LZ transitions in a cavity field originate from the photon-number-dependent Rabi frequency. The asymmetric sequential LZ dynamics of Bose-condensed atoms in a cavity field may open up a way to explore the fundamental many-body physics in coupled atom-photon systems.
\end{abstract}

DOI: 10.1103/PhysRevA.94.023618

\section{INTRODUCTION}

The great achievements in manipulating and probing ultracold atoms in a cavity offer a new platform for exploring many-body systems and their dynamics. Up to now, the strong atom-cavity coupling has been experimentally demonstrated in several laboratories by using an atomic Bose-Einstein condensate (BEC) [1-6]. The BEC-cavity experiments can be used to study the coupled atom-photon system with both large and small atom and photon numbers [1-4]. The BEC-cavity system provides an ideal platform for exploring exotic many-body quantum effects, such as quantum phase transition [5-12], many-body quantum entanglement [13-15], precision measurement [16-18], quantum optomechanics [19$22]$ and many-body quantum dynamics $[23,24]$. On the other hand, as an important fundamental problem, the many-body Landau-Zener (LZ) tunneling with BECs has attracted much attention for a long time [25-28]. However, the many-body LZ tunneling in the BEC-cavity system, in particular, how the cavity field affects the LZ dynamics of atoms, is still unclear.

Due to the atom-atom interaction, the many-body LZ tunneling is very different from the single-particle one. The sequential LZ tunneling induced by interaction blockade have been found in the Bose-Josephson system (BJS) [29,30], the Bose-Hubbard ladder [31] and spinor BECs within optical superlattices [32]. For an example, in a BJS [33-36], due to the interplay between the intermode bias and the atom-atom interaction, the resonant tunneling and interaction blockade take place $[29,30]$. There appears a population ladder indicating a series of interaction blockades. The variance of the

*lichaoh2@mail.sysu.edu.cn; chleecn@gmail.com relative population would exhibit several peaks representing the single-atom resonant tunneling. Due to the fixed Josephson coupling in such a BJS, the step slopes of the ladder and the resonant tunneling peaks are symmetric about the zero bias (which corresponds to the zero detuning).

In a BEC-cavity system, due to the quantization nature of the cavity field, the picture of the sequential LZ dynamics may have significant differences. In comparison with the BJS, the Rabi frequency in a BEC-cavity system acts the role of the Josephson coupling. Unlike the Josephson coupling, the Rabi frequency is no longer fixed and it is proportional to the square root of the cavity-photon number [37]. In the case of few photons, as the photon number changes in the LZ process, the Rabi frequency dramatically changes when the detuning is tuned. Furthermore, if there are insufficient photons to excite all the atoms to the upper level, an incomplete sequential $\mathrm{LZ}$ process would occur. Thus, in addition to the atom-atom interaction and the detuning, the photon number also plays an important role in the LZ process of the BEC-cavity system. Simultaneously, during the time evolution, the atoms may also have an influence on the cavity field. Therefore, it is of great interest to investigate (i) how the sequential LZ dynamics is affected by the cavity field and (ii) how the cavity field changes during such a LZ process.

In this article, we study the LZ process of an ensemble of interacting Bose-condensed atoms trapped in a cavity. In the framework of second quantization, the BEC-cavity system can be described by a modified Tavis-Cummings model with an additional nonlinear term determined by the atom-atom interaction [24,38]. If the initial cavity field is described by a Fock state, the BEC-cavity system only involves a single excitation number. In the adiabatic sweeping of the detuning, the interplay between the detuning and the 


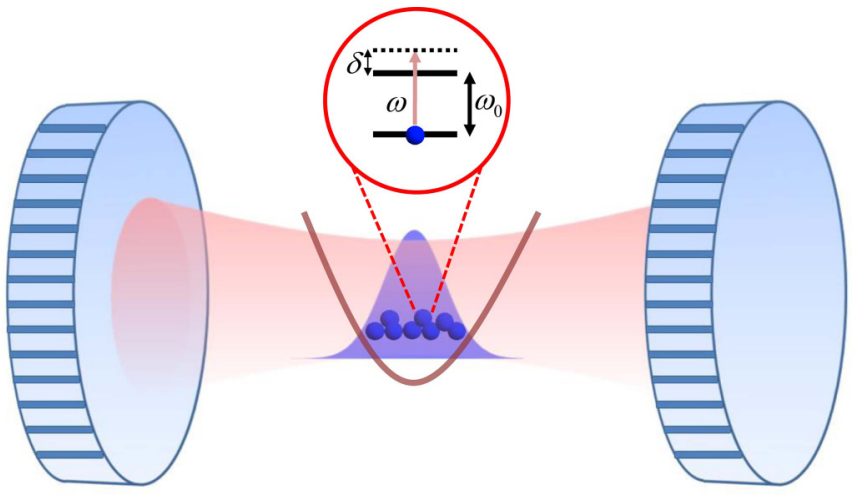

FIG. 1. Sketch of trapped interacting Bose-condensed atoms in an optical cavity. Each atom is identically coupled to the cavity mode with the coupling strength $g / \sqrt{N_{a}}$. Here, the frequencies of the cavity field and the atomic transition are denoted by $\omega$ and $\omega_{0}$, respectively. The detuning is $\delta=\omega-\omega_{0}$ and the effective atom-atom interaction is characterized by $E_{c}$.

atom-atom interaction results in the asymmetric sequential LZ transitions, in which the atoms absorb or emit photons one by one and the sequential LZ transitions are asymmetric. If the initial cavity field is described by a coherent state, the BEC-cavity system will involve multiple excitation numbers. Different from the case of single excitation numbers, in the asymmetric sequential LZ process, the atoms may no longer absorb entirely one photon for every population step. The number of absorbed and/or emitted photons becomes less than 1 and it gradually decreases even in the adiabatic limit. During the $\mathrm{LZ}$ process, the cavity field is no longer a coherent state and its intermittent collapse and revival appear.

This article is organized as follows. In Sec. II, we describe the model and give its Hamiltonian. In Sec. III, we investigate the asymmetric sequential LZ process involving single excitation numbers. In Sec. IV, we consider the atoms initially coupled with a coherent cavity field and then study the asymmetric sequential LZ process involving multiple excitation numbers. We derive an analytical formula for sequential ladders of the photon number and the relative atom number. The adiabaticity conditions for the sequential LZ transitions are also analytically estimated. An intriguing finding of fractional steps is observed when the average number is small compared with atom number. In addition, we analyze the effects of the cavity photon loss on the sequential LZ dynamics. The phenomenon of collapses and revivals of the cavity field is also revealed. Finally, in Sec. V, we give a brief summary and discussion.

\section{MODEL}

We consider an ensemble of two-state Bose-condensed atoms confined in an optical cavity. The schematic diagram is shown in Fig. 1. As in the experiments of ${ }^{87} \mathrm{Rb}$ atoms $[1,2]$, the two internal states can be chosen as $5{ }^{2} S_{1 / 2}|F=2\rangle$ and $5^{2} P_{3 / 2}\left|F^{\prime}=3\right\rangle$, where the transition is at $\lambda=780.2 \mathrm{~nm}$. The atoms are coupled with the cavity mode, in which one atom in the lower level may absorb a photon and jump to the upper level, or may be taken from the upper level into the lower level with a photon emitted. The Bose-condensed atoms are confined in a sufficiently deep dipole trap so that all the atoms may only occupy the lowest motional state. Assuming that the coupling fields are spatially uniform and that the atom-atom collision does not change the internal states. Each atom identically couples with a single-mode cavity field [39]. Therefore, the system obeys a second quantized Hamiltonian within a single-mode approximation (in the unit of $\hbar=1$ throughout the paper):

$$
\begin{aligned}
H= & \omega \hat{a}^{\dagger} \hat{a}+\sum_{j=\uparrow, \downarrow}\left(E_{0 j} \hat{b}_{j}^{\dagger} \hat{b}_{j}+\frac{1}{2} G_{j j} \hat{b}_{j}^{\dagger} \hat{b}_{j}^{\dagger} \hat{b}_{j} \hat{b}_{j}\right) \\
& +G_{\uparrow \downarrow} \hat{b}_{\downarrow}^{\dagger} \hat{b}_{\uparrow}^{\dagger} \hat{b}_{\uparrow} \hat{b}_{\downarrow}+\frac{g}{\sqrt{N_{a}}}\left(\hat{a} \hat{b}_{\uparrow}^{\dagger} \hat{b}_{\downarrow}+\hat{a}^{\dagger} \hat{b}_{\downarrow}^{\dagger} \hat{b}_{\uparrow}\right),
\end{aligned}
$$

where $\omega$ is the frequency of cavity mode, $N_{a}=N_{\uparrow}+N_{\downarrow}=$ $\hat{b}_{\uparrow}^{\dagger} \hat{b}_{\uparrow}+\hat{b}_{\downarrow}^{\dagger} \hat{b}_{\downarrow}$ is the atom number, $E_{0 j}$ is the energy of the atoms in state $|j\rangle, G_{j j}$ is the atom-atom interaction energies of state $|j\rangle, G_{\uparrow \downarrow}$ is the atom-atom interaction energy between states $|\uparrow\rangle$ and $|\downarrow\rangle$, and $g$ is the homogenous coupling strength between the cavity mode and the Bose-condensed atoms.

Regarding all the atoms as spin- $\frac{1}{2}$ particles, one can define the angular momentum operators as

$$
\begin{gathered}
\hat{J}_{x}=\frac{\hat{b}_{\uparrow}^{\dagger} \hat{b}_{\downarrow}+\hat{b}_{\uparrow} \hat{b}_{\downarrow}^{\dagger}}{2}, \\
\hat{J}_{y}=\frac{\hat{b}_{\uparrow}^{\dagger} \hat{b}_{\downarrow}-\hat{b}_{\uparrow} \hat{b}_{\downarrow}^{\dagger}}{2 i}, \\
\hat{J}_{z}=\frac{\hat{b}_{\uparrow}^{\dagger} \hat{b}_{\uparrow}-\hat{b}_{\downarrow}^{\dagger} \hat{b}_{\downarrow}}{2} .
\end{gathered}
$$

The system has two good quantum numbers: the atom number $N_{a}$ and the excitation number $N_{e}=\hat{a}^{\dagger} \hat{a}+N_{\uparrow}=$ $\hat{a}^{\dagger} \hat{a}+\hat{J}_{z}+\frac{N_{a}}{2}$, satisfying the commutation $\left[N_{a}, H\right]=0$ and $\left[N_{e}, H\right]=0$. Here, when the excitation number is much larger than the atom number, i.e., $N_{e} \gg N_{a}$, it corresponds to the large excitation number condition, which is close to the classical field limit. However, if the excitation number is comparable with or smaller than the atom number, i.e., $N_{e} \lesssim$ $N_{a}$, it refers to the small excitation number condition. The quantization of the cavity mode would make a difference with a classical field and the photon number may affect the properties of the whole system. We carefully compare the results under large and small excitation conditions in the following.

Since $N_{a}$ and $N_{e}$ are conserved quantities, the constant terms $O\left(N_{a}\right)$ and $O\left(N_{a}^{2}\right)$ can be eliminated and the Hamiltonian becomes

$$
H=\omega_{0} \hat{J}_{z}+\omega \hat{a}^{\dagger} \hat{a}+\frac{E_{c}}{2} \hat{J}_{z}^{2}+\frac{g}{\sqrt{N_{a}}}\left(\hat{a}^{\dagger} \hat{J}_{-}+\hat{a} \hat{J}_{+}\right),
$$

where $\hat{J}_{ \pm}=\hat{J}_{x} \pm i \hat{J}_{y}$ are the raising and lowering operators of atoms, the atomic transition frequency $\omega_{0}=E_{0 \uparrow}-E_{0 \downarrow}+$ $\frac{1}{2}\left(N_{a}-1\right)\left(G_{\uparrow \uparrow}-G_{\downarrow \downarrow}\right)$, and the effective atom-atom interaction $E_{c}=G_{\uparrow \uparrow}+G_{\downarrow \downarrow}-2 G_{\uparrow \downarrow}$. Further, shifting the zero point of the Hamiltonian (5), one gets

$$
H=-\frac{\delta}{2} \hat{J}_{z}+\frac{\delta}{2} \hat{a}^{\dagger} \hat{a}+\frac{E_{c}}{2} \hat{J}_{z}^{2}+\frac{g}{\sqrt{N_{a}}}\left(\hat{a}^{\dagger} \hat{J}_{-}+\hat{a} \hat{J}_{+}\right),
$$

with the detuning $\delta=\omega-\omega_{0}$. According to the last term, for fixed $g$ and $N_{a}$, the Rabi frequency is proportional to the square 
root of the photon number (i.e., $\Omega \propto \sqrt{n}$ ), which is changeable with respect to the state of the cavity field.

The state of the system can be expanded as

$$
|\psi\rangle=\sum_{n, m} C_{n, m}|n, m\rangle,
$$

where $n$ is the photon number of the Fock state, $m=\left(N_{\uparrow}-\right.$ $\left.N_{\downarrow}\right) / 2$ is the relative atom number, and $C_{n, m}$ are the coefficients of basis $|n, m\rangle$. Due to the conservation of the excitation number, when $N_{e}<N_{a}$, the number of bases can be reduced to $N_{e}+1$, and the state of the system can be expanded as

$$
|\psi\rangle=\sum_{l=0}^{N_{e}} C_{l}^{N_{e}}\left|N_{e}-l,-\frac{N_{a}}{2}+l\right\rangle .
$$

For $N_{e} \geqslant N_{a}$, only $N_{a}+1$ bases are enough, and the state of the system can be expanded as

$$
|\psi\rangle=\sum_{l=0}^{N_{a}} C_{l}^{N_{e}}\left|N_{e}-l,-\frac{N_{a}}{2}+l\right\rangle .
$$

Here, $C_{l}^{N_{e}}$ in Eqs. (8) and (9) denotes the coefficient with $N_{e}-l$ photons and $l$ atoms populating in the upper level.

\section{LANDAU-ZENER PROCESS INVOLVING SINGLE EXCITATION NUMBERS}

First, we consider the system with fixed single excitation numbers and study the static properties of the system under small and large excitation number conditions. Starting from Hamiltonian (6), we choose atom number $N_{a}=10$ and investigate the situations with the excitation numbers $N_{e}=5$, $N_{e}=10$, and $N_{e}=1000$. Here, the system state $|\Psi\rangle$ can be expressed according to Eq. (8) or Eq. (9). We vary $\delta$ from negative detuning to positive detuning and obtain the corresponding ground states. The detuning $\delta$ tends to populate the atoms into the upper level while the repulsive atom-atom interaction $\left(E_{c}>0\right)$ tends to populate the atoms equally in both levels. For far negative detuning, i.e., $\omega \ll \omega_{0}$ and $\delta \rightarrow-\infty$, the ground state is $\left|N_{e},-\frac{N_{a}}{2}\right\rangle$, which corresponds to all atoms being in the lower level and the photon number equals the excitation number $N_{e}$. For far positive detuning, i.e., $\omega \gg \omega_{0}$ and $\delta \rightarrow \infty$, the form of the ground state is related to the excitation number. When $N_{e} \geqslant N_{a}$, the ground state is $\left|N_{e}-N_{a}, \frac{N_{a}}{2}\right\rangle$, which is the state in which all atoms occupy the upper level and the photon number becomes $N_{e}-N_{a}$. When $N_{e}<N_{a}$, it becomes $\left|0,-\frac{N_{a}}{2}+N_{e}\right\rangle$, where only a portion of atoms occupy the upper level and no photons remain. In other regions of detuning, the ground state depends sensitively on the parameters $g, \delta$, and $E_{c}$. To better reveal the properties of the system, the expectations of the photon number $\langle n\rangle=\left\langle\psi\left|\hat{a}^{\dagger} \hat{a}\right| \psi\right\rangle$ and the half relative atom number $\left\langle N_{\uparrow}-N_{\downarrow}\right\rangle / 2=\left\langle\psi\left|\hat{J}_{z}\right| \psi\right\rangle$ and the variance of relative atom number $\operatorname{Var}\left(N_{\uparrow}-N_{\downarrow}\right)=4\left(\left\langle\hat{J}_{z}^{2}\right\rangle-\left\langle\hat{J}_{z}\right\rangle^{2}\right)$ for the ground state are calculated and shown in Fig. 2.

\section{A. Asymmetric sequential population ladders}

There is a series of ladderlike steps for the half relative atom number. These sequential population ladders are induced by the atom-atom interaction. The steps become steeper and the plateaus get more smooth when the atom-atom interaction becomes larger. The height of every step equals exactly 1 . For every step, one of the atoms in the lower level can absorb one photon and be excited to the upper level. The appearance of sequential population ladders is similar to the interaction blockade observed in the Bose-Josephson junctions [29,30]. For a two-component Bose-Einstein condensate linked by classical Raman fields, the competition between detuning and nonlinear interaction will result in a sequential population ladder for the relative atom number, where the slopes of the steps are exactly symmetric about $\delta=0$.

However, for the two-state Bose-condensed atoms coupled with a quantized cavity mode, the number of photons makes the shape of the ladders significantly different. When the excitation number is smaller than the atom number, $N_{e}<N_{a}$, the structure is totally asymmetric since there are not enough photons to excite all the atoms and the population ladder ceases to increase when there are no photons inside the cavity [see Fig. 2(a)]. In the situation of $N_{e}=10$, the step slopes of the population ladders are also no longer symmetric about $\delta=0$. For the same magnitude of detuning $|\delta|$, the step slope under negative detuning, $-\delta$, is always less sharper than the one under positive detuning, $+\delta$.

The asymmetry of the step slopes comes from the changing Rabi frequency during the sequential LZ processes. Adiabatically sweeping the detuning from negative to positive, the photon number decreases downstairs from $N_{e}$ to 0 . Therefore, the Rabi frequency $\Omega$ is getting smaller. The sharpness is determined by the ratio between the Rabi frequency and the atom-atom interaction $\Omega / E_{c}$, where the smaller $\Omega / E_{c}$ corresponds to the sharper step slope. Specially, when the photon number decreases to 0 , the Rabi frequency vanishes, and no transitions happen. Since the photon number under negative detuning, $-\delta$, is always larger than the one under positive detuning, $+\delta$, the step slopes of the ladder for $\left\langle N_{\uparrow}-N_{\downarrow}\right\rangle / 2$ are asymmetric about $\delta=0$. The first and the last step slopes have the biggest difference because they, respectively, correspond to the largest and the smallest photon numbers [see the insets of Fig. 2(c)]. For other pairs of step slopes, the difference is smaller.

As the excitation number increases, the photon number difference under $-\delta$ and $+\delta$ becomes less dramatic and the Rabi frequency $\Omega$ tends to be uniform. For a large excitation number, $N_{e}=1000$, the step slopes of the ladder become almost symmetric about $\delta=0$ [see the insets of Fig. 2(e)]. This is because when the photon number is extremely large compared with the atom number, the cavity field can be treated as a classical field with a fixed complex number, and the sequential behaviors would return to the symmetric one in a BJS.

\section{B. Asymmetric variance of relative atom number}

For further investigation, we look in the variance of relative atom number $\operatorname{Var}\left(N_{\uparrow}-N_{\downarrow}\right)$. There are a series of peaks for $\operatorname{Var}\left(N_{\uparrow}-N_{\downarrow}\right)$. The locations of the peaks correspond to the locations of the step slopes in the ladder of $\left\langle N_{\uparrow}-N_{\downarrow}\right\rangle / 2$. The stronger atom-atom interaction $E_{c}$ induces sharper and narrower peaks, which is consistent with the sharpness of 

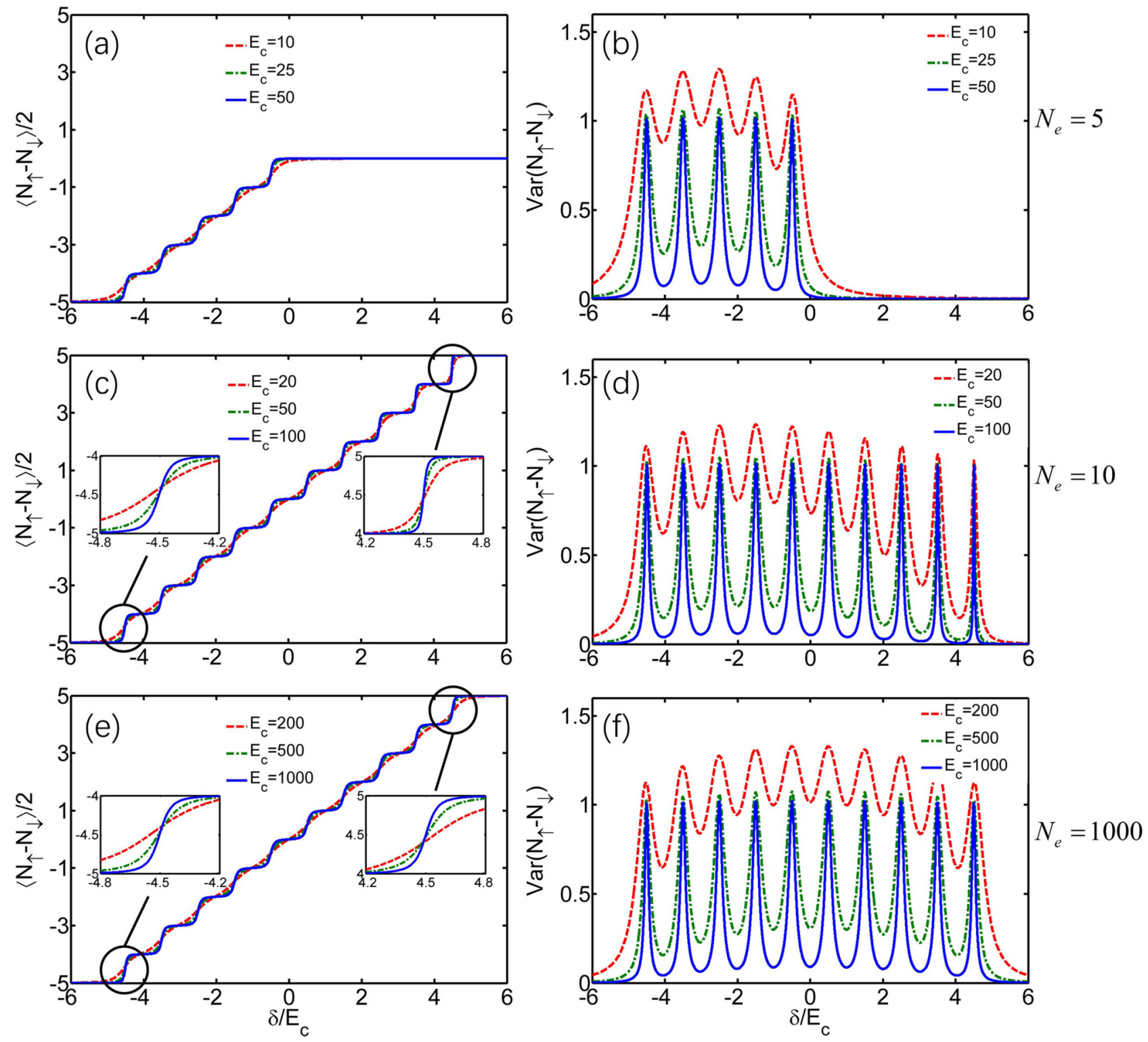

FIG. 2. Static properties of the system involving single excitation numbers. The top, middle, and bottom rows of panels correspond to the cases of $N_{e}=5, N_{e}=10$, and $N_{e}=1000$, respectively. The left and right columns represent the expectations of the half relative atom number $\left\langle N_{\uparrow}-N_{\downarrow}\right\rangle / 2=\left\langle\hat{J}_{z}\right\rangle$ and the variance of the relative atom number $\operatorname{Var}\left(N_{\uparrow}-N_{\downarrow}\right)=4\left(\left\langle\hat{J}_{z}^{2}\right\rangle-\left\langle\hat{J}_{z}\right\rangle^{2}\right)$ versus detuning, respectively. Here, $N_{a}=10$ and $g=1$.

the step slopes. When $N_{e}=5$, the peaks are completely asymmetric. Several peaks appear when $\delta<0$ and no peaks appear when $\delta>0$ [see Fig. 2(b)].

The asymmetry is also obvious for $N_{e}=10$ [see Fig. 2(d)]. The total structure is asymmetric about $\delta=0$. The width and the height of the first and the last peaks are totally different. The last peak is much sharper than the first one, which is in accordance with the situation of the step slopes of the population ladder. The plateaus in the ladder of $\left\langle N_{\uparrow}-N_{\downarrow}\right\rangle / 2$ indicate the interaction blockades. In the regions of the plateaus, the system is approximately in the state $|n, m\rangle$ with a definite photon number and a relative atom number. Therefore, the variance of the relative atom number is suppressed. The resonance peaks are caused by the quasidegeneracy between the two states $\mid N_{e}-k+1,-\frac{N_{a}}{2}+$ $k-1\rangle$ and $\left|N_{e}-k,-\frac{N_{a}}{2}+k\right\rangle$, with $k=1, \ldots, \min \left(N_{a}, N_{e}\right)$ in the neighboring plateaus. In the intermediate region of the step slopes, the superposition of these two states leads to $\operatorname{Var}\left(N_{\uparrow}-N_{\downarrow}\right)$ increasing dramatically. One can treat the atom-photon coupling term as a perturbation $[29,30]$ and obtain the locations of the peaks with $\delta / E_{c}=l+1 / 2$ for $l=$ $\left\{-N_{a} / 2,-N_{a} / 2+1, \ldots, N_{a} / 2-1\right\}$, which is in agreement with the numerical results.

More importantly, since the Rabi frequency becomes weaker when the photon number decreases, the relative atom number also shows asymmetric properties. When the Rabi frequency decreases, the perturbation term becomes smaller, and the two neighboring states $\left|N_{e}-k+1,-\frac{N_{a}}{2}+k-1\right\rangle$ and $\left|N_{e}-k,-\frac{N_{a}}{2}+k\right\rangle$ are closer to degeneracy. Therefore, the variance of the relative atom number would occur narrower 
and sharper peaks at the degeneracy location. However, for a large excitation number, the asymmetry becomes much smaller. Under $N_{e}=1000$, the structure of the variance $\operatorname{Var}\left(N_{\uparrow}-N_{\downarrow}\right)$ becomes more symmetric about $\delta=0$ [see Fig. 2(f)].

The set of peaks that indicate the single-atom resonance transition can be used to design the single-atom device. For this system, varying the photon number in the cavity can adjust the sensitivity of the response and the atom-atom interaction need not be too strong, which can be promising for application in sensitive detection and high-precision metrology.

\section{LANDAU-ZENER DYNAMICS INVOLVING MULTIPLE EXCITATION NUMBERS}

In the following, we consider the ensemble of interacting Bose-condensed atoms coupled with a cavity field in a coherent state. The coherent state of photons is natural and it is easily prepared in experiments. We sweep the detuning to investigate the LZ dynamics of the system. Initially, the atoms are all prepared in the lower level. The cavity field is a coherent state, which is the eigenstate of the annihilation operator $\hat{a}$ and can be written as $|\alpha\rangle=e^{-\frac{|\alpha|^{2}}{2}} \sum_{n=0}^{\infty} \frac{\alpha^{n}}{\sqrt{n !}}|n\rangle$, with $|\alpha|^{2}$ being the average photon number of the coherent state. Therefore, the initial state of the whole system can be expressed as

$$
|\Psi(0)\rangle=\left|\alpha,-\frac{N_{a}}{2}\right\rangle=\sum_{N_{e}=0}^{\infty} w_{N_{e}}\left|N_{e},-\frac{N_{a}}{2}\right\rangle,
$$

which involves multiple excitation numbers with $w_{N_{e}}=$ $e^{-\frac{|\alpha|^{2}}{2}} \frac{\alpha^{N_{e}}}{\sqrt{N_{e} !}}$. During the sweeping, the time evolution of the system state $|\Psi(t)\rangle$ obeys the Schrödinger equation,

$$
i \frac{\partial|\Psi(t)\rangle}{\partial t}=H(t)|\Psi(t)\rangle,
$$

where $H(t)$ is the Hamiltonian (5) with the time-dependent detuning $\delta(t)$. The system state can be expanded as

$$
|\Psi(t)\rangle=\sum_{N_{e}=0}^{\infty} w_{N_{e}}\left(\sum_{l} C_{l}^{N_{e}}(t)\left|N_{e}-l,-\frac{N_{a}}{2}+l\right\rangle\right),
$$

where $C_{l}^{N_{e}}(t)$ is the time-dependent coefficient of the basis in subspace with the excitation number $N_{e}$. There are $N_{e}$ subspaces with different excitation numbers and the bases of each subspace depend on the excitation numbers. Each subspace with different $N_{e}$ is orthogonal and decoupled from others. Therefore, the dynamics in each subspace is independent and we can deal with the time-evolution problem individually and then sum up together according to the weight factor of each subspace $w_{N_{e}}$. In addition, the average excitation number of the system is always conserved and equals the initial average photon number.

In our calculation, we fix the atom number $N_{a}=4$, the coupling strength $g=1$, and atom-atom interaction $E_{c}=$ 100. Meanwhile, the detuning $\delta$ is linearly swept according to $\delta(t)=\delta_{0}+\beta t$ with the initial detuning $\delta_{0}<0$ and the sweeping rate $\beta=\frac{2\left|\delta_{0}\right|}{\tau}$, where $\tau$ is the total sweeping time. Here, we use $\beta$ to characterize the nonadiabaticity of the sweeping. The smaller sweeping rate $\beta$ refers to a slower sweeping of detuning, which means a greater probability of staying in the instantaneous ground state when the LZ process occurs. The properties of the LZ dynamics with multiple excitation numbers may differ according to the distribution of the excitation numbers which is related to the average photon number of the initial coherent state. Here, for the initial coherent states, we choose the average photon number to be $|\alpha|^{2}=4$ and $|\alpha|^{2}=25$.

\section{A. Asymmetric sequential Landau-Zener dynamics}

The evolution of the photon number, the variance of the relative atom number, and the half relative atom number versus the detuning $\delta(t)$ for different sweeping rates $\beta$ are shown in Fig. 3. The three observables of the evolved state can be, respectively, calculated according to

$$
\begin{aligned}
\langle n(t)\rangle & =\left\langle\Psi(t)\left|\hat{a}^{\dagger} \hat{a}\right| \Psi(t)\right\rangle \\
& =\sum_{N_{e}, l}\left(N_{e}-l\right)\left|w_{N_{e}} C_{l}^{N_{e}}(t)\right|^{2}, \\
\frac{\left\langle N_{\uparrow}(t)-N_{\downarrow}(t)\right\rangle}{2} & =\left\langle\Psi(t)\left|\hat{J}_{z}\right| \Psi(t)\right\rangle \\
& =\sum_{N_{e}, l}\left(-\frac{N_{a}}{2}+l\right)\left|w_{N_{e}} C_{l}^{N_{e}}(t)\right|^{2},
\end{aligned}
$$

and

$$
\begin{aligned}
\operatorname{Var}\left[N_{\uparrow}(t)-N_{\downarrow}(t)\right] & \\
= & 4\left[\left\langle\Psi(t)\left|\hat{J}_{z}^{2}\right| \Psi(t)\right\rangle-\left(\left\langle\Psi(t)\left|\hat{J}_{z}\right| \Psi(t)\right\rangle\right)^{2}\right] \\
= & 4\left\{\sum_{N_{e}, l}\left(-\frac{N_{a}}{2}+l\right)\right)^{2}\left|w_{N_{e}} C_{l}^{N_{e}}(t)\right|^{2} \\
& \left.-\left[\sum_{N_{e}, l}\left(-\frac{N_{a}}{2}+l\right)\left|w_{N_{e}} C_{l}^{N_{e}}(t)\right|^{2}\right]^{2}\right\} .
\end{aligned}
$$

For $|\alpha|^{2}=4$, where the average photon number equals the atom number, the population ladder for the half relative atom number coincides with the one in the adiabatic limit when the sweeping rate is sufficiently small (e.g., $\beta=2$ ) [see Fig. 3(a)]. When the sweeping becomes fast (e.g., $\beta=10$ ), the population ladder oscillates and the heights of the steps become less obvious. The sequential LZ transitions gradually disappear when the nonadiabatic effect is strong enough for extremely fast driving (e.g., $\beta=100$ ). For $|\alpha|^{2}=25$, where the average photon number is much greater than the atom number, the sequential LZ dynamics versus the nonadiabatic effect is similar [see Fig. 3(b)]. However, it does not require such slow driving to attain its adiabatic limit (e.g., $\beta=20$ is slow enough while for $|\alpha|^{2}=4$ it is not). That is because when the average photon number increases, the Rabi frequency and the gaps of the avoided energy level crossings become larger. The analytical analysis for the adiabaticity condition is given in the next subsection.

The variances of the relative atom number for small and large $|\alpha|^{2}$ are very different [see Figs. 3(c) and 3(d)]. For the adiabatic sweeping with $|\alpha|^{2}=25$, the variance $\operatorname{Var}\left(N_{\uparrow}-N_{\downarrow}\right)$ is similar to the one with a large single excitation number shown in Fig. 2(f). For the adiabatic sweeping with $|\alpha|^{2}=4$, 

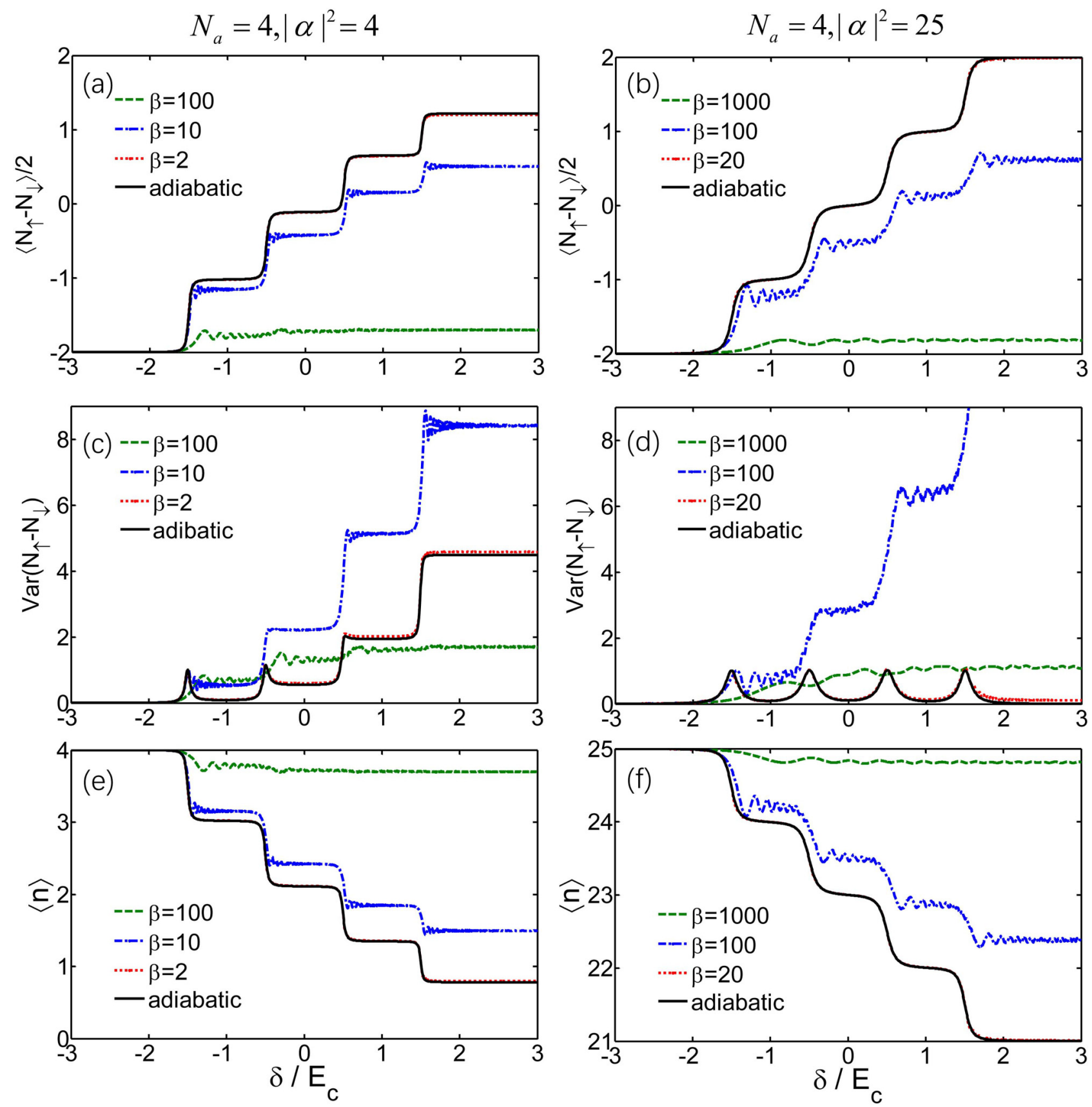

FIG. 3. Dynamical evolution of the half relative atom number, the variance of relative atom number, and the photon number. The atom number $N_{a}=4$ and the initial cavity field is in a coherent state with average photon numbers $|\alpha|^{2}=4$ and $|\alpha|^{2}=25$. The detuning is linearly swept according to $\delta(t)=\delta_{0}+\beta t$. The black solid lines are the adiabatic limit and the other lines are numerical results with different sweeping rates $\beta$. In order to ensure the initial detuning is large enough, we choose $\delta_{0}=-3 E_{c}$ with $E_{c}=100$ for calculation.

the variance $\operatorname{Var}\left(N_{\uparrow}-N_{\downarrow}\right)$ shows two peaks for the first and second LZ transitions and then shows a ladderlike structure with unequal step heights. If the sequential LZ transitions for small $N_{e}$ are incomplete, the atomic state is a superposition state of different $m=\left(N_{\uparrow}-N_{\downarrow}\right) / 2$ and the corresponding variance $\operatorname{Var}\left(N_{\uparrow}-N_{\downarrow}\right)$ becomes large. Thus, as the detuning increases, the system is dominated by the incomplete sequential LZ transitions for small $N_{e}$, and the atomic number variance changes from peaks to ladderlike steps. However, for large $|\alpha|^{2}$, the atomic state in every subspace changes synchronously and the variance appears as a series of peaks due to the quasidegeneracy.

When taking the nonadiabatic effects into account, the variances change dramatically. For both $|\alpha|^{2}=4$ and $|\alpha|^{2}=$ 25 , under a very slow sweeping, the variances approach their adiabatic limits. Under intermediate sweeping, the atomic state becomes a superposition state of different $m=\left(N_{\uparrow}-\right.$ $\left.N_{\downarrow}\right) / 2$ after every LZ transition. As the LZ transitions occur sequentially, more different $m=\left(N_{\uparrow}-N_{\downarrow}\right) / 2$ components accumulate and induce the variance $\operatorname{Var}\left(N_{\uparrow}-N_{\downarrow}\right)$ to increase 
in ladderlike steps [e.g., the blue dash-dotted lines in Figs. 3(c) and 3(d)]. For the fast sweeping, the system states hardly follow the instantaneous ground state and change slightly and the variances change more smoothly.

More interestingly, the sequential population ladders for small average photon numbers exhibit another kind of asymmetric property. The heights (but not the slopes) of the steps are obviously unequal and become fractional [see Figs. 3(a) and 3(e)]. For $|\alpha|^{2}=4$, even though in the adiabatic limit, the height of each step is less than 1 and different from others. The relative atom population ladder increases upstairs with the height of the steps gradually becomeing smaller and smaller. The four photons on average could not be entirely absorbed by the four atoms even after four times of LZ transitions.

In contrast, for $|\alpha|^{2}=25$ in the adiabatic limit, the population ladder of the half relative atom number increases upstairs with each step's height equal to nearly 1 , which is similar to the LZ process with single excitation numbers shown in Fig. 2. The coherent state with a large average photon number can be approximately treated as a classical field. When the average photon number is large enough, the initial atoms in the lower level could gradually absorb the photon one by one through every LZ transition [see Figs. 3(b) and 3(f)].

It is worth mentioning that, when $\delta(t)$ is swept adiabatically to 0 , the evolved atomic state becomes a twin Fock state, in which the populations in the upper and lower levels are exactly equal $\left(\left\langle N_{\uparrow}-N_{\downarrow}\right\rangle=0\right)$ and the variance of the relative atom number $\operatorname{Var}\left(N_{\uparrow}-N_{\downarrow}\right)=0$. The atomic twin Fock state is a highly entangled state that can be used to implement quantum metrology $[40,41]$. In our system, preparing the initial atomic state in $\left|-\frac{N_{a}}{2}\right\rangle$ and the cavity photon field in a coherent state with large $|\alpha|^{2}$, an atomic twin Fock state can be generated via an adiabatic linear sweeping from negative detuning to 0 . This offers a scheme for quantum state engineering in highly entangled state preparation.

\section{B. Analytical analysis for sequential Landau-Zener dynamics}

To explore the sequential LZ dynamics, we derive an analytical formula for ladders of the photon number and the relative atom number. In our model, the whole Hilbert space can be divided into several decoupled subspaces and we can investigate the dynamics individually in every subspace and finally sum them up together. For a subspace with excitation number $N_{e}$ and atom number $N_{a}$, LZ transitions would respectively occur $N_{a}$ and $N_{e}$ times for $N_{e} \geqslant N_{a}$ and $N_{e}<N_{a}$. The $k$ th LZ transition happens between the states $\left|N_{e}-k+1,-\frac{N_{a}}{2}+k-1\right\rangle$ and $\left|N_{e}-k,-\frac{N_{a}}{2}+k\right\rangle$, with $k=1, \ldots, \min \left(N_{a}, N_{e}\right)$. Therefore, the sequential LZ transitions in a subspace can be treated as a sequence of conventional two-level LZ transitions. In a conventional LZ transition with a sweeping rate $\beta$ with the minimum gap $\Delta$ for its avoided energy level crossing, and starting from its ground state at time $t \rightarrow-\infty$, the probability of finding the system remaining in the ground state at time $t \rightarrow+\infty$ is given by the LZ formula $[42,43]$

$$
P(\Delta, \beta)=1-\exp \left(-\frac{\pi \Delta^{2}}{2 \beta}\right)
$$

By applying the conventional two-level LZ formula to each avoided energy level crossing, the probability of staying in the instant ground state at the $k$ th LZ transition reads

$$
P\left(\Delta_{k}^{N_{e}}, \beta\right)=1-\exp \left[-\frac{\pi\left(\Delta_{k}^{N_{e}}\right)^{2}}{2 \beta}\right],
$$

where $\Delta_{k}$ denotes the minimum gap of the $k$ th LZ transition, which corresponds to the energy gap at $\delta_{k} / E_{c}=-\left(N_{a}+\right.$ 1) $/ 2+k$, with $k=1,2, \ldots, \min \left(N_{a}, N_{e}\right)$. The $k$ th minimum gap is proportional to the atom-photon coupling term of the Hamiltonian (6), and it can be expressed in the form of

$$
\Delta_{k}^{N_{e}}=2 g \sqrt{\frac{k\left(N_{a}-k+1\right)\left(N_{e}-k+1\right)}{N_{a}}} .
$$

By applying the LZ formula (17) one by one, we can obtain the final probability of staying in the instant ground state after $k$ times of $\mathrm{LZ}$ transitions, which is given as

$$
P_{N_{e}, k}(\beta)=\prod_{l=1}^{k} P\left(\Delta_{l}^{N_{e}}, \beta\right) .
$$

For every LZ transition, the probability of remaining in the ground state also corresponds to the probability of absorbing one photon. From this point, we can figure out the heights of every population step of the photon number during the sweeping. The height of the $k$ th step equals exactly $P_{N_{e}, k}(\beta)$. Therefore, the photon number of the plateaus in the ladder can be obtained analytically:

$$
\langle n\rangle_{N_{e}}= \begin{cases}N_{e}, & \delta<\delta_{1}, \\ N_{e}-\sum_{m=1}^{\min \left(k, N_{e}\right)} P_{N_{e}, m}(\beta), & \delta_{k} \leqslant \delta<\delta_{k+1}, \\ N_{e}-\sum_{m=1}^{\min \left(N_{e}, N_{a}\right)} P_{N_{e}, m}(\beta), & \delta \geqslant \delta_{N_{a}},\end{cases}
$$

Correspondingly, the half relative atom number can be expressed as

$$
\begin{aligned}
& \frac{\left\langle N_{\uparrow}-N_{\downarrow}\right\rangle_{N_{e}}}{2} \\
& \quad= \begin{cases}-\frac{N_{a}}{2}, & \delta<\delta_{1}, \\
-\frac{N_{a}}{2}+\sum_{m=0}^{\min \left(k, N_{e}\right)} P_{N_{e}, m}(\beta), & \delta_{k} \leqslant \delta<\delta_{k+1}, \\
-\frac{N_{a}}{2}+\sum_{m=0}^{\min \left(N_{e}, N_{a}\right)} P_{N_{e}, m}(\beta), & \delta \geqslant \delta_{N_{a}},\end{cases}
\end{aligned}
$$

where $\delta_{k}=\left[-\left(N_{a}+1\right) / 2+k\right] E_{c}$ and the index $k=$ $1,2, \ldots, \min \left(N_{a}, N_{e}\right)$. Given the sweeping rate $\beta$, one can figure out the population ladders analytically. In Fig. 4, we plot the population ladders of the photon numbers for $N_{e}=\{1,2, \ldots, 10\}$ under sweeping rates $\beta=\{10,100\}$. The dashed lines are the numerical results while the solid lines are obtained analytically according to Eq. (20). It is obvious that the analytical population ladders are consistent with the numerical ones. Since the minimum gap $\Delta_{k}$ increases with the excitation number $N_{e}$, it needs slower driving for smaller $N_{e}$. For fast sweeping $\beta=100$, the step height decreases obviously when $N_{e}$ changes from 10 to 1 , while for slow sweeping $\beta=10$ the step heights for most $N_{e}$ approach 1 . Therefore, for small $|\alpha|^{2}$, the dominated $N_{e}$ is small and requires slower sweeping to access the adiabaticity condition. 
(a) $\beta=100$

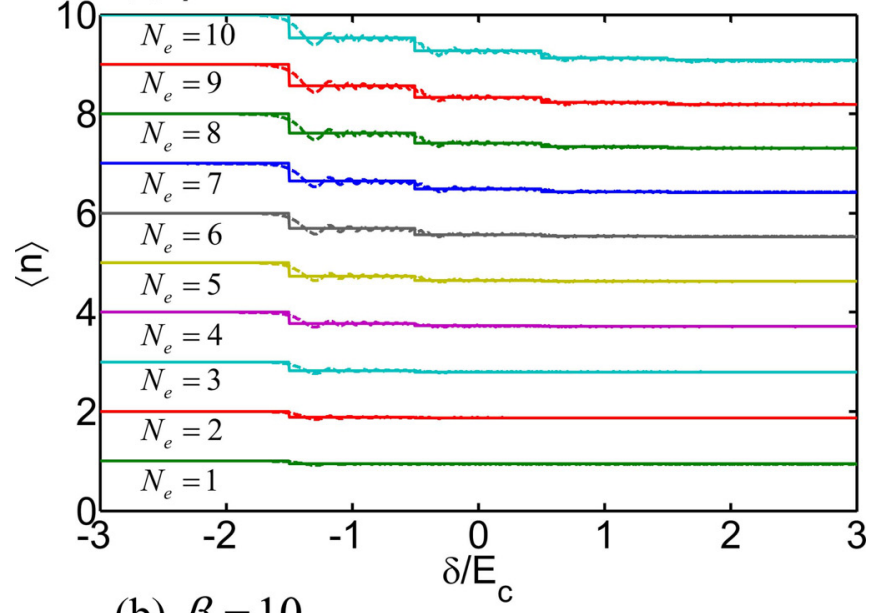

(b) $\beta=10$

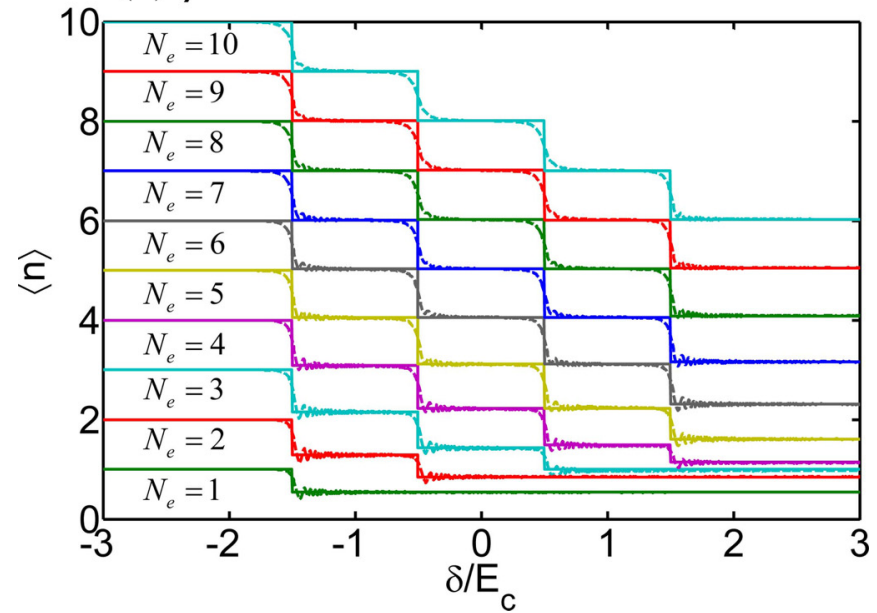

FIG. 4. The sequential LZ dynamics for photon numbers in subspaces under different sweeping rates $\beta$. Here, $N_{e}$ are chosen from 1 to $10, N_{a}=4, g=1$, and $E_{c}=100$. The solid lines are obtained analytically according to Eq. (20). The dashed lines are the numerical results.

By summing up the results of all subspaces, the average photon number and the half relative atom number of the system are given as

$$
\langle n\rangle=\sum_{N_{e}=0}^{\infty} w_{N_{e}}^{2}\langle n\rangle_{N_{e}}
$$

and

$$
\frac{\left\langle N_{\uparrow}-N_{\downarrow}\right\rangle}{2}=\sum_{N_{e}=0}^{\infty} w_{N_{e}}^{2} \frac{\left\langle N_{\uparrow}-N_{\downarrow}\right\rangle_{N_{e}}}{2} .
$$

In our system, the adiabatic condition can be estimated approximately according to

$$
\frac{\left[\min _{k}\left(\Delta_{k}^{|\alpha|^{2}-\min \left(\left.\alpha\right|^{2}, N_{a}\right)+1}\right)\right]^{2}}{2 \beta} \gtrsim 2 .
$$

For $|\alpha|^{2}=4, \beta \lesssim 1$; for $|\alpha|^{2}=25, \beta \lesssim 21$. This estimation can be confirmed by the comparison with numerical calculations [see Fig. 3].
One of the interesting findings is the appearance of fractional steps in population ladders for small $|\alpha|^{2}$ even under adiabatic sweeping. This peculiar phenomenon results from the property of the coherent state with few average photon numbers. The initial coherent state is a superposition of the infinite Fock state including the vacuum state with different weight coefficients $w_{N_{e}}$. For small $N_{e}$, there are insufficient photons to excite all the atoms to the upper level, and this incomplete sequential LZ process would contribute to the fractional steps if the weight coefficient $w_{N_{e}}$ were not ignorable.

Below we give an analytical explanation for the fractional steps. When the sweeping is adiabatic $(\beta \rightarrow 0)$, all $P_{N_{e}, k}(\beta) \rightarrow$ 1 and so the average photon number in subspace of $N_{e}$ becomes

$$
\langle n\rangle_{N_{e}}= \begin{cases}N_{e}, & \delta<\delta_{1}, \\ N_{e}-\min \left(k, N_{e}\right), & \delta_{k} \leqslant \delta<\delta_{k+1}, \\ N_{e}-\min \left(N_{e}, N_{a}\right), & \delta \geqslant \delta_{N_{a}} .\end{cases}
$$

According to Eq. (22), the average photon number of the system can be obtained by

$$
\langle n\rangle= \begin{cases}\sum_{N_{e}=0}^{\infty} w_{N_{e}}^{2} N_{e}, & \delta<\delta_{1}, \\ \sum_{N_{e}=k}^{\infty} w_{N_{e}}^{2}\left(N_{e}-k\right), & \delta_{k} \leqslant \delta<\delta_{k+1}, \\ \sum_{N_{e}=N_{a}}^{\infty} w_{N_{e}}^{2}\left(N_{e}-N_{a}\right), & \delta \geqslant \delta_{N_{a}} .\end{cases}
$$

Using the condition $\sum_{N_{e}=0}^{\infty} w_{N_{e}}^{2} N_{e}=|\alpha|^{2}$, we can simplify Eq. (26) to

$\langle n\rangle= \begin{cases}|\alpha|^{2}, & \delta<\delta_{1}, \\ |\alpha|^{2}-k-\sum_{N_{e}=0}^{k-1} w_{N_{e}}^{2}\left(N_{e}-k\right), & \delta_{k} \leqslant \delta<\delta_{k+1}, \\ |\alpha|^{2}-N_{a}-\sum_{N_{e}=0}^{N_{a}-1} w_{N_{e}}^{2}\left(N_{e}-N_{a}\right), & \delta \geqslant \delta_{N_{a}} .\end{cases}$

From Eq. (27), we can further obtain the $k$ th step height of the photon number (as well as the relative atom number):

$$
h_{k}=1-\sum_{N_{e}=0}^{k-1} w_{N_{e}}^{2} \text {. }
$$

As a result, for small $|\alpha|^{2}$, the weight coefficients $w_{N_{e}}^{2}$ of small $N_{e}$ are dominated, which leads to the fractional steps. For example, $|\alpha|^{2}=4, w_{0}^{2}=0.0183, w_{1}^{2}=0.0733, w_{2}^{2}=0.1465$, and $w_{3}^{2}=0.1954$ and we can get $h_{1}=0.9817, h_{2}=0.9084$, $h_{3}=0.7619$, and $h_{4}=0.5665$. The results are the same with the numerical findings. Moreover, for large $|\alpha|^{2}$, the weight coefficients $w_{N_{e}}^{2}$ of small $N_{e}$ are nearly 0 , and the height of every step equals 1 . Since the total excitation number of the system is conserved, the explanation is also valid for the relative atom number.

\section{Dissipative sequential Landau-Zener dynamics}

In cavity QED experiments, the system would suffer decoherence induced by the escape of photons out of the cavity or the decay of the atoms without emitting photons $[44,45]$. These decoherence effects may affect the sequential LZ transitions. In this subsection, we mainly discuss the effect of dissipation (cavity photon loss) on the sequential LZ dynamics. During the sweeping, the cavity photons may escape from the 

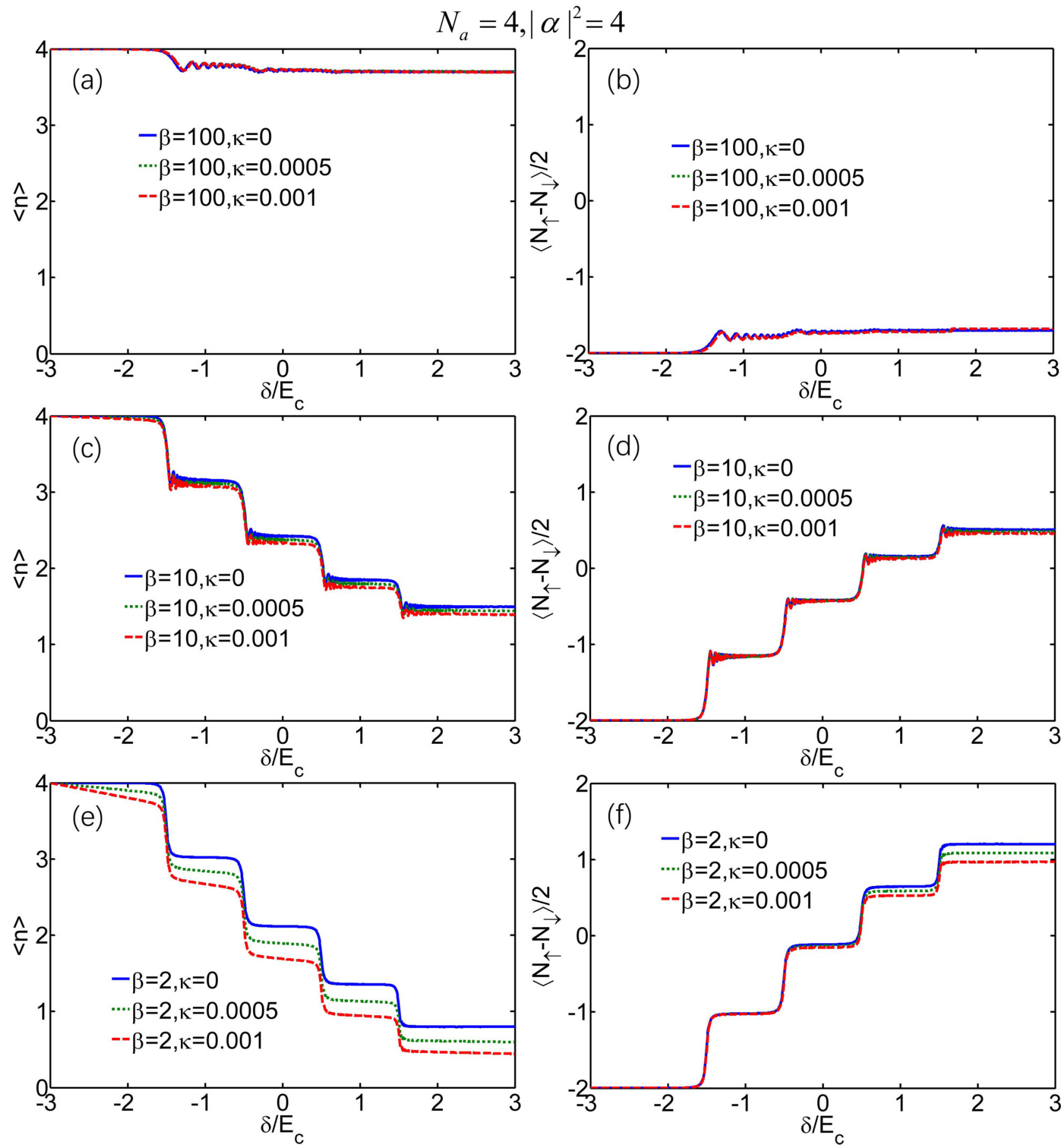

FIG. 5. The sequential LZ dynamics under cavity photon losses. The influences of damping rates $\kappa$ on the photon number and the relative atom number under different sweeping rates $\beta$ are shown. Here, the parameters are chosen as $N_{a}=4,\left|\alpha^{2}\right|=4, g=1$, and $E_{c}=100$.

cavity and the total excitation number would no longer be conserved. Therefore, it is necessary to describe the system state by the reduced density matrix $\rho=|\tilde{\Psi}\rangle\langle\tilde{\Psi}|$, where the bases of $|\tilde{\Psi}\rangle$ include different total excitation numbers and differ from the ones in Eq. (12). The sequential dynamics under the cavity photon loss can be characterized by the Lindblad master equation [44-46]:

$$
\frac{\partial \rho}{\partial t}=-i[H(t), \rho]+L_{\kappa}[\rho]
$$

with

$$
L_{\kappa}[\rho]=\frac{\kappa}{2}\left(2 \hat{a} \rho \hat{a}^{\dagger}-\hat{a}^{\dagger} \hat{a} \rho-\rho \hat{a}^{\dagger} \hat{a}\right)
$$

where $\kappa$ is the cavity photon loss rate.

In our calculation, the reduced density matrix of the system is broken up into different density matrices $\rho_{N_{e}}$ in subspaces. Then we solve the master equation (29) independently and calculate the observable expectation by $\left\langle\hat{O}_{N_{e}}\right\rangle=\operatorname{Tr}\left(\hat{O} \rho_{N_{e}}\right)$ and sum up together according to $\langle\hat{O}\rangle=w_{N_{e}}^{2}\left\langle\hat{O}_{N_{e}}\right\rangle$. The time 

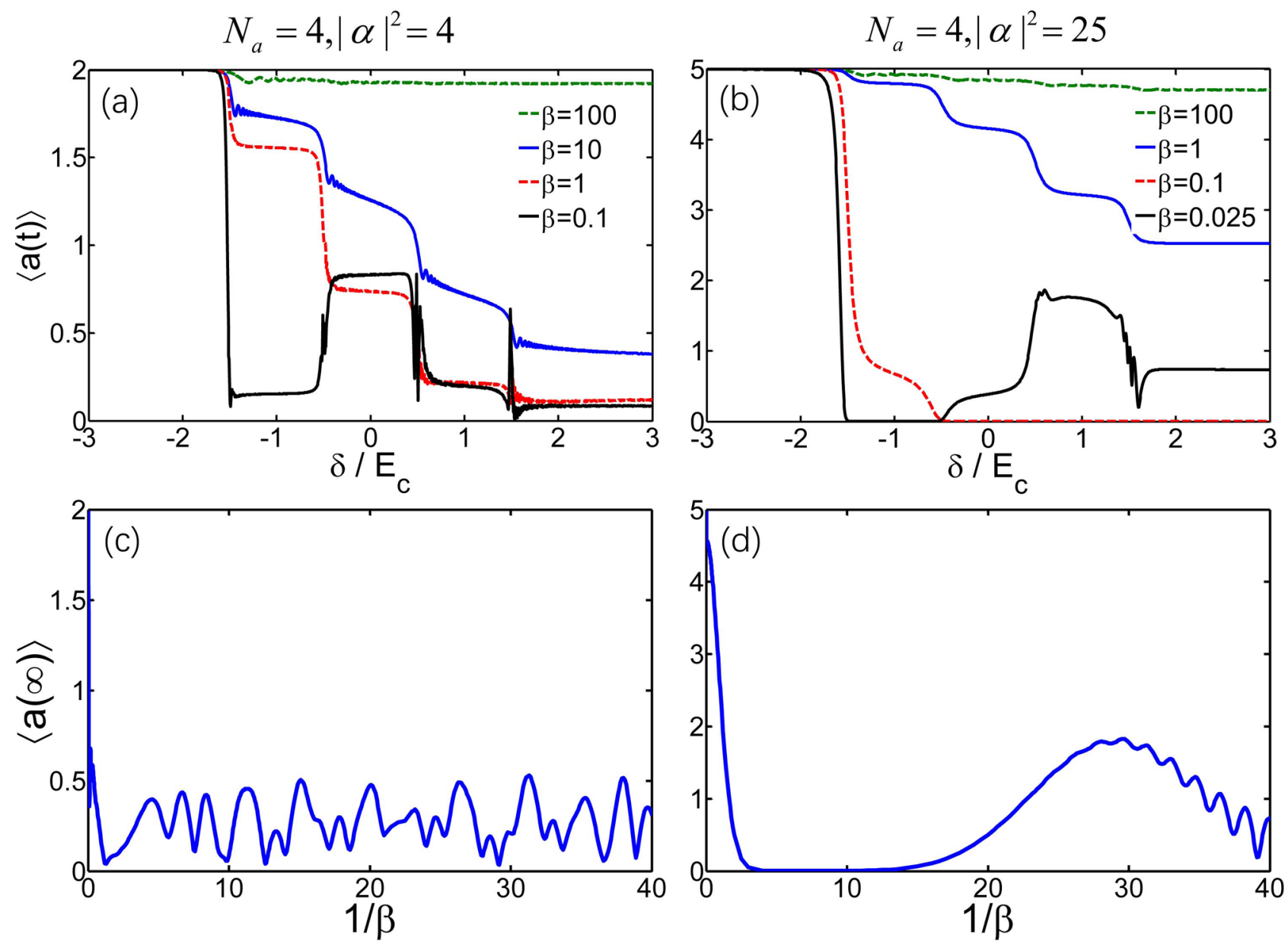

FIG. 6. Collapses and revivals of cavity field coherence. The evolution of the cavity field coherence $\langle a(t)\rangle$ during the LZ dynamics with different sweeping rates $\beta$ for (a) $|\alpha|^{2}=4$ and (b) $|\alpha|^{2}=25$. The final cavity field coherence $\langle a(\infty)\rangle$ versus the inverse of the sweeping rate $1 / \beta$ for (c) $|\alpha|^{2}=4$ and (d) $|\alpha|^{2}=25$. Here, $N_{a}=4, g=1$, and $E_{c}=100$.

evolutions of the photon number and the half relative atom number under dissipation for $|\alpha|^{2}=4$ are shown in Fig. 5.

For $\kappa=0$, it returns to the ideal case, where the total excitation number is conserved. For $\kappa>0$, the influences of the dissipation become obvious when the sweeping rates get smaller. Given $\beta=100$ and $\beta=10$, the influences of dissipation are very small and the sequential LZ dynamics are almost unchanged [see Figs. 5(a)-5(d)].

When the sweeping becomes slower, the total evolution time becomes longer and more photon losses accumulate. For $\beta=2$, which approaches the adiabatic limit for $|\alpha|^{2}=4$, although the cavity photon loss decreases the photon number, it only changes the step heights of the fractional ladders slightly [see Fig. 5(e)]. The cavity photon loss affects the ladder of the half relative atom number gradually, but the step heights shrink only a little during the whole process [see Fig. 5(f)].

It is shown that the cavity photon loss may also affect the sequential population ladders. Obviously, the sequential population ladders will disappear if the number of lost photons is large compared with $|\alpha|^{2}$. However, if the number of lost photons is small compared with $|\alpha|^{2}$, which may be realized in the strong coupling regime $[44,45]$, the fractional steps of the half relative atom number can still be observed.

\section{Collapse and revivals of cavity field coherence}

We further investigate how the coherence of the cavity field varies during the LZ processes with different sweeping rates. Since the evolution of each photon Fock state will accumulate a phase dependent on the photon number, the phase differences between different Fock states will also play an important role during the LZ dynamics. In order to study the effect of this phase difference during the evolution, a common quantity to measure is the expectation of the photon field annihilation operator [46]:

$$
\begin{aligned}
\langle a(t)\rangle & =\langle\Psi(t)|\hat{a}| \Psi(t)\rangle \\
& =\sum_{N_{e}, l} \sqrt{N_{e}-l} w_{N_{e}-1}^{*} w_{N_{e}} C_{l}^{N_{e}-1 *}(t) C_{l}^{N_{e}}(t),
\end{aligned}
$$

which well describes the coherence of the cavity field. The coherence of the photon field $\langle a(t)\rangle$ versus the evolution time $t$ with different sweeping rates $\beta$ are shown in Figs. 6(a) and $6(\mathrm{~b})$. The dynamical behaviors of $\langle a(t)\rangle$ are much different than the those of the photon number $\langle n(t)\rangle$.

When $|\alpha|^{2}=25$, for very fast sweeping $\beta=100$, the coherence of the cavity field $\langle a(t)\rangle$ changes slightly. For modest sweeping $\beta=1,\langle a(t)\rangle$ oscillates in ladder shape. For slower sweeping $\beta=0.1,\langle a(t)\rangle$ drops dramatically and remains 0 
when passing through all the LZ transitions. However, for very slow sweeping $\beta=0.025,\langle a(t)\rangle$ drops rapidly to 0 when the first LZ transition happens and begins to increase and oscillate after the second LZ transition. Finally, the coherence remains a certain value after all the LZ transitions. This is the collapse and revival of the cavity field coherence due to the long-time accumulated phase difference between different Fock state components of the coherent state.

The change of the cavity field coherence for $|\alpha|^{2}=4$ is similar to that for $|\alpha|^{2}=25$. For fast sweeping, the coherence of the photon field $\langle a(t)\rangle$ changes slightly with small oscillations. For modest sweeping, $\langle a(t)\rangle$ oscillates in a downstairs ladderlike shape. For very slow sweeping, the coherence of the cavity field $\langle a(t)\rangle$ first collapses and then revives. It drops quickly at the first $\mathrm{LZ}$ transition point and oscillates a lot afterwards until all the LZ transitions are finished. However, for $|\alpha|^{2}=4$, the coherence of the cavity field seldom decreases to 0 and the oscillations are more dramatic compared with the ones for $|\alpha|^{2}=25$.

The coherence of the cavity field will tend to a steady value after all the LZ transitions and the final coherence $\langle a(\infty)\rangle$ depends on the sweeping rate. The dependence of $\langle a(\infty)\rangle$ on the inverse sweeping rate $1 / \beta$ is shown in Figs. $6(\mathrm{c})$ and $6(d)$. Obviously, the final coherence of the cavity field versus the inverse sweeping rate also exhibits the behavior of collapses and revivals. For $|\alpha|^{2}=25$, with fast sweeping, the collapse occurs as the difference between different Fock states grows, leading to a destructive interference. With slower sweeping, the coherence drops to death and remains 0 for a wide range. For much slower sweeping, the phase differences grow back in phase and the coherence gradually begins to revive. However, the revival will oscillate and finally cease for extremely slow sweeping. For $|\alpha|^{2}=4$, the coherence changes more rapidly. The final coherence of the cavity field drops dramatically at first but revives quickly and then begins to oscillate in disorder around a modest range. It may be seen that the cavity field coherence for a small average photon number would not exhibit obvious collapses and revivals, which is different from the cavity field coherence for a large average photon number.

\section{SUMMARY AND DISCUSSIONS}

In summary, we have explored the phenomenon of the asymmetric sequential LZ dynamics in an ensemble of interacting two-level Bose-condensed atoms trapped in an optical cavity. Instead of directly coupling two atomic states with an optical cavity mode, the Hamiltonian (5) may also be effectively achieved in a $\Lambda$-type three-level configuration where an external microwave field is applied to drive the transition between two internal atomic states $[18,47,48]$. The features of Bose-condensed atoms in a cavity with a small photon number are extremely different from the those of Bose-condensed atoms in a cavity with a large photon number. For a relatively small photon number, the interplay between the detuning and the atom-atom interaction leads to asymmetric sequential LZ transitions. There appears to be asymmetric interaction blockade and single-atom resonance tunneling when the LZ process involves only a single excitation number, which is mainly due to the photon-number-dependent Rabi frequency. The single-atom resonance may be used to design high-precision devices and sensitive detectors.

Instead of an initial Fock state, considering an initial coherent cavity field with a small average photon number, an asymmetric population ladder with unequal fractional height steps is observed. The asymmetric sequential LZ dynamics of the system are also studied. We derive an analytical formula for sequential population ladders. The adiabaticity conditions for the sequential LZ transitions are analytically estimated. The intriguing finding of fractional steps is also explained. In addition, we analyze the effects of the cavity dissipation on the sequential LZ dynamics. On the other hand, the state of the cavity field changes dramatically during the time-evolution process. The behaviors of collapses and revivals of the cavity field coherence are revealed.

Further, for an initial coherent state with a large average cavity-photon number, the sequential LZ dynamics in a cavity approach the sequential LZ dynamics in a continuous laser field. Starting from all atoms in the lower energy level, the atoms can absorb photons one by one in the sequential LZ process if the detuning is swept sufficiently slowly. When the detuning is swept to 0 , an atomic twin Fock state can be generated. In addition, the photon state is no longer a coherent state and its coherence changes dramatically. This may be associated with the generation of photon-deleted coherent states [49], which is totally nonclassical. Similarly, if one sweeps the detuning oppositely, and the atoms are initially in the upper level, there would be the phenomenon that the atoms emit photons one by one during the sequential LZ process. This may be related to the creation of the photon-added coherent states [50]. Different from most previous schemes which add or subtract only a single photon, our scheme can add or subtract the desired number of photons by dynamically controlling the detuning. This indicates that it may provide a tool for preparing nonclassical photon states and also may be applied to demonstrate the bosonic commutation relations [51-53].

\section{ACKNOWLEDGMENTS}

The authors thank B. Lu and F. Mei for useful discussions and suggestions. This work is supported by the National Basic Research Program of China (NBRPC) under Grant No. 2012CB821305 and the National Natural Science Foundation of China (NNSFC) under Grants No. 11374375, No. 11465008, and No. 11574405.
[1] F. Brennecke, T. Donner, S. Ritter, T. Bourdel, M. Köhl, and T. Esslinger, Cavity QED with a Bose-Einstein condensate, Nature (London) 450, 268 (2007).

[2] Y. Colombe, T. Steinmetz, G. Dubois, F. Linke, D. Hunger, and J. Reichel, Strong atom-field coupling for Bose-Einstein condensates in an optical cavity on a chip, Nature (London) 450, 272 (2007).

[3] J. Goldwin, M. Trupke, J. Kenner, A. Ratnapala, and E. A. Hinds, Fast cavity-enhanced atom detection with low noise and high fidelity, Nat. Commun. 2, 418 (2011). 
[4] W. Chen, J. Hu, Y. Duan, B. Braverman, H. Zhang, and V. Vuletić, Carving Complex Many-Atom Entangled States by Single-Photon Detection, Phys. Rev. Lett. 115, 250502 (2015).

[5] K. Baumann, R. Mottl, F. Brennecke, and T. Esslinger, Exploring Symmetry Breaking at the Dicke Quantum Phase Transition, Phys. Rev. Lett. 107, 140402 (2011).

[6] H. Ritsch, P. Domokos, F. Brennecke, and T. Esslinger, Cold atoms in cavity-generated dynamical optical potentials, Rev. Mod. Phys. 85, 553 (2013).

[7] I. B. Mekhov, C. Maschler, and H. Ritsch, Probing quantum phases of ultracold atoms in optical lattices by transmission spectra in cavity quantum electrodynamics, Nat. Phys. 3, 319 (2007).

[8] J. Larson, B. Damski, G. Morigi, and M. Lewenstein, MottInsulator States of Ultracold Atoms in Optical Resonators, Phys. Rev. Lett. 100, 050401 (2008).

[9] D. Nagy, G. Kónya, G. Szirmai, and P. Domokos, Dicke-Model Phase Transition in the Quantum Motion of a Bose-Einstein Condensate in an Optical Cavity, Phys. Rev. Lett. 104, 130401 (2010).

[10] K. Baumann, C. Guerlin, F. Brennecke, and T. Esslinger, Dicke quantum phase transition with a superfluid gas in an optical cavity, Nature (London) 464, 1301 (2010).

[11] G. Vacanti, S. Pugnetti, N. Didier, M. Paternostro, G. M. Palma, R. Fazio, and V. Vedral, Photon Production from the Vacuum Close to the Superradiant Transition: Linking the Dynamical Casimir Effect to the Kibble-Zurek Mechanism, Phys. Rev. Lett. 108, 093603 (2012).

[12] M. R. Bakhtiari, A. Hemmerich, H. Ritsch, and M. Thorwart, Nonequilibrium Phase Transition of Interacting Bosons in an Intra-Cavity Optical Lattice, Phys. Rev. Lett. 114, 123601 (2015).

[13] F. Haas, J. Volz, R. Gehr, J. Reichel, and J. Estève, Entangled states of more than 40 atoms in an optical fiber cavity, Science 344, 180 (2014).

[14] L. Sun, A. Petrenko, Z. Leghtas, B. Vlastakis, G. Kirchmair, K. M. Sliwa, A. Narla, M. Hatridge, S. Shankar, J. Blumoff, L. Frunzio, M. Mirrahimi, M. H. Devoret, and R. J. Schoelkopf, Tracking photon jumps with repeated quantum non-demolition parity measurements, Nature (London) 511, 444 (2014).

[15] R. McConnell, H. Zhang, J. Hu, S. Ćuk, and V. Vuletić, Entanglement with negative Wigner function of almost 3,000 atoms heralded by one photon, Nature (London) 519, 439 (2015).

[16] M. Zuppardo, J. P. Santos, G. De Chiara, M. Paternostro, F. L. Semiao, and G. M. Palma, Cavity-aided quantum parameter estimation in a bosonic double-well Josephson junction, Phys. Rev. A 91, 033631 (2015).

[17] P. Hamilton, M. Jaffe, J. M. Brown, L. Maisenbacher, B. Estey, and H. Müller, Atom Interferometry in an Optical Cavity, Phys. Rev. Lett. 114, 100405 (2015).

[18] A. B. Bhattacherjee and D. Sharma, Spin squeezing and quantum Fisher information in the Jaynes-Cummings Dicke model, arXiv:1607.01930.

[19] A. Dalafi, M. H. Naderi, M. Soltanolkotabi, and Sh. Barzanjeh, Nonlinear effects of atomic collisions on the optomechanical properties of a Bose-Einstein condensate in an optical cavity, Phys. Rev. A 87, 013417 (2013).
[20] D. Nagy, G. Szirmai, and P. Domokos, Cavity optomechanics with a trapped, interacting Bose-Einstein condensate, Eur. Phys. J. D 67, 124 (2013).

[21] M. Aspelmeyer, T. J. Kippenberg, and F. Marquardt, Cavity optomechanics, Rev. Mod. Phys. 86, 1391 (2014).

[22] K. A. Yasir and W. Liu, Tunable bistability in hybrid BoseEinstein condensate optomechanics, Sci. Rep. 5, 10612 (2015).

[23] A. Alvermann, L. Bakemeier, and H. Fehske, Collapse-revival dynamics and atom-field entanglement in the nonresonant Dicke model, Phys. Rev. A 85, 043803 (2012).

[24] B. M. Garraway, The Dicke model in quantum optics: Dicke model revisited, Philos. Trans. R. Soc., A 369, 1137 (2011).

[25] B. Wu and Q. Niu, Nonlinear Landau-Zener tunneling, Phys. Rev. A 61, 023402 (2000).

[26] Y. Chen, S. D. Huber, S. Trotzky, I. Bloch, and E. Altman, Manybody Landau-Zener dynamics in coupled one-dimensional Bose liquids, Nat. Phys. 7, 61 (2011).

[27] Y. Qian, M. Gong, and C. Zhang, Many-body Landau-Zener transition in cold-atom double-well optical lattices, Phys. Rev. A 87, 013636 (2013).

[28] H. Zhong, Q. Xie, J. Huang, X. Qin, H. Deng, J. Xu, and C. Lee, Photon-induced sideband transitions in a many-body LandauZener process, Phys. Rev. A 90, 023635 (2014).

[29] C. Lee, L.-B. Fu, and Y. S. Kivshar, Many-body quantum coherence and interaction blockade in Josephson-linked BoseEinstein condensates, Europhys. Lett. 81, 60006 (2008).

[30] P. Cheinet, S. Trotzky, M. Feld, U. Schnorrberger, M. MorenoCardoner, S. Fölling, and I. Bloch, Counting Atoms Using Interaction Blockade in an Optical Superlattice, Phys. Rev. Lett. 101, 090404 (2008).

[31] H. Deng, H. Dai, J. Huang, X. Qin, J. Xu, H. Zhong, C. He, and C. Lee, Cluster Gutzwiller study of the Bose-Hubbard ladder: Ground-state phase diagram and many-body Landau-Zener dynamics, Phys. Rev. A 92, 023618 (2015).

[32] A. Wagner, C. Bruder, and E. Demler, Spin-1 atoms in optical superlattices: Single-atom tunneling and entanglement, Phys. Rev. A 84, 063636 (2011).

[33] R. Gati and M. K. Oberthaler, A bosonic Josephson junction, J. Phys. B 40, R61 (2007).

[34] C. Lee, Universality and Anomalous Mean-Field Breakdown of Symmetry-Breaking Transitions in a Coupled Two-Component Bose-Einstein Condensate, Phys. Rev. Lett. 102, 070401 (2009).

[35] C. Gross, T. Zibold, E. Nicklas, J. Estève, and M. K. Oberthaler, Nonlinear atom interferometer surpasses classical precision limit, Nature (London) 464, 1165 (2010).

[36] C. Lee, J. Huang, H. Deng, H. Dai, and J. Xu, Nonlinear quantum interferometry with Bose condensed atoms, Front. Phys. 7, 109 (2012).

[37] J. M. Fink, M. Göppl, M. Baur, R. Bianchetti, P. J. Leek, A. Blais, and A. Wallraff, Climbing the Jaynes-Cummings ladder and observing its $\sqrt{n}$ nonlinearity in a cavity QED system, Nature (London) 454, 315 (2008).

[38] M. Feng, Y. P. Zhong, T. Liu, L. L. Yan, W. L. Yang, J. Twamley, and $\mathrm{H}$. Wang, Exploring the quantum critical behavior in a driven Tavis-Cummings circuit, Nat. Commun. 6, 7111 (2015).

[39] J. Lee, G. Vrijsen, I. Teper, O. Hosten, and M. A. Kasevich, Many-atom cavity QED system with homogeneous atom cavity coupling, Opt. Lett. 39, 4005 (2014). 
[40] B. Lücke, M. Scherer, J. Kruse, L. Pezzé, F. Deuretzbacher, P. Hyllus, O. Topic, J. Peise, W. Ertmer, J. Arlt, L. Santos, A. Smerzi, and C. Klempt, Twin matter waves for interferometry beyond the classical limit, Science 334, 773 (2011).

[41] C. C. Gerry and J. Mimih, Heisenberg-limited interferometry with pair coherent states and parity measurements, Phys. Rev. A 82, 013831 (2010).

[42] L. Landau, Phys. Z. Sowjetunion 2, 46 (1932).

[43] C. Zener, Non-adiabatic crossing of energy levels, Proc. R. Soc. London, Ser. A 137, 696 (1932).

[44] A. Reiserer and G. Rempe, Cavity-based quantum networks with single atoms and optical photons, Rev. Mod. Phys. 87, 1379 (2015).

[45] M. J. Hartmann, F. G. S. L. Brandão, and M. B. Plenio, Quantum many-body phenomena in coupled cavity arrays, Laser Photonics Rev. 2, 527 (2008).

[46] J. Keeling and V. Gurarie, Collapse and Revivals of the Photon Field in a Landau-Zener Process, Phys. Rev. Lett. 101, 033001 (2008).

[47] Y. Li, P. Zhang, and Z. D. Wang, Extended JC-Dicke model for two-component atomic BEC inside a cavity, Eur. Phys. J. D 58, 379 (2010).
[48] S. Li, L. Fu, and J. Liu, Adiabatic geometric phase for a BoseEinstein condensate coupled to a cavity, Phys. Rev. A 84, 053610 (2011).

[49] J. Honer, R. Löw, H. Weimer, T. Pfau, and H. P. Büchler, Artificial Atoms Can Do More Than Atoms: Deterministic Single Photon Subtraction from Arbitrary Light Fields, Phys. Rev. Lett. 107, 093601 (2011).

[50] N. Lee, H. Benichi, Y. Takeno, S. Takeda, J. Webb, E. Huntington, and A. Furusawa, Teleportation of nonclassical wave packets of light, Science 332, 330 (2011).

[51] A. Zavatta, S. Viciani, and M. Bellini, Quantum-to-classical transition with single-photon-added coherent states of light, Science 306, 660 (2004).

[52] V. Parigi, A. Zavatta, M. Kim, and M. Bellini, Probing quantum commutation rules by addition and subtraction of single photons to/from a light field, Science 317, 1890 (2007)

[53] A. Zavatta, V. Parigi, M. S. Kim, and M. Bellini, Subtracting photons from arbitrary light fields: Experimental test of coherent state invariance by single-photon annihilation, New J. Phys. 10, 123006 (2008). 